\title{
A Method for the Localization of Damage in a CFRP Plate using Damping
}

\author{
D. Montalvão a,*, A.M.R. Ribeiro ${ }^{\mathrm{b}}$, J. Duarte-Silva ${ }^{\mathrm{a}}$
}

${ }^{a}$ Department of Mechanical Engineering, Escola Superior de Tecnologia de Setúbal, Instituto Politécnico de Setúbal, Campus do IPS, Estefanilha, 2910-761 Setúbal, Portugal

${ }^{\mathrm{b}}$ Department of Mechanical Engineering, Instituto Superior Técnico, Universidade Técnica de Lisboa, Av. Rovisco Pais, 1049-001 Lisbon, Portugal

\begin{abstract}
In the past 25 years, the scientific and industrial communities have made big efforts on the fields of Damage Detection and Structural Health Monitoring (SHM). However, no single approach has proven appropriate for all situations. Composite materials, which are receiving an increasing attention in the aeronautical industry, namely Carbon Fibre Reinforced Plastics (CFRP), are very sensitive to impacts of medium and low energy. Typically, Barely Visible Impact Damage (BVID) will occur, constituting an unsafe failure of difficult assessment. To assess (detect, locate and quantify) damage in this kind of material is still a challenge, especially if a huge amount of sensors or expensive equipments at hand are not used. In this work, a methodology that makes use of a reduced amount of conventional sensors is explored, with the aim of locating damage for a low cost on components that are subjected to impacts during service. This represents a considerable benefit, namely for the assessment of damage in aeronautical components, compared to most methods used today. This work can also give a major contribution to the research community since uncommon approaches will be used to model damage in composite materials, namely the modal damping factor as the main feature for damage localization.
\end{abstract}

Keywords: Structural Health Monitoring; Damage; Delamination; Damping; Composite; Vibration.

\section{Introduction}

Vibration-based Structural Health Monitoring (SHM) and damage detection are fields of study that are

\footnotetext{
* Corresponding author. Tel.: +351 265790 000; fax: +351 265721869 .

E-mail addresses: dmontalvao@est.ips.pt (D. Montalvão), aribeiro@ dem.ist.utl.pt (A.M.R. Ribeiro), jsilva@est.ips.pt (J. Duarte-Silva).
} 
arising great interest. Bonfiglioli et al. [1] argue that monitoring the health state of infrastructures seems to be one of the largest industries in the world. Important advances in this field until 2002 are described in Doebling et al. [2, 3] and Sohn et al. [4]. More recently, Montalvão et al. [5] presented a review on vibration-based SHM with special emphasis on composite materials. Other reviews may bring insight in more specific branches of damage detection and SHM [6-9].

The use of Fibre Reinforced Plastics (FRP) is experiencing an increased growth, namely in the aeronautical, naval and automotive industries, because of their excellent mechanical properties in conjunction with their low weight and easy shaping. Nevertheless, composite materials are very different from metals with respect to their particular failure modes, which may be in the form of matrix cracking, fibre breakage, interlaminar delamination, etc $[10,11]$. Also, the extreme sensitivity of composite materials to impact loads constitutes a hindrance to its utilization.

For aeronautical structures, a field where this problem has been quite studied, the components have to undergo (i) low energy impacts caused by dropped tools or mishandling during assembly and maintenance, (ii) medium energy impacts caused in-service by foreign objects such as stones or birds and, (iii) in military aircraft, high energy impacts caused by weaponry projectiles [12].

In a low energy impact (but high enough to produce damage), only a very small indentation will be seen on the impact surface. This level of damage is often referred to as Barely Visible Impact Damage (BVID). Generally, Carbon Fibre Reinforced Plastics (CFRP) are very sensitive to medium and low energy impacts. Matrix cracks will appear and interact, leading to the delamination process. Moreover, on the opposite side of the impact, it is possible that fibre breakage will occur.

Cawley and Adams [13] give a formulation for damage detection, localization and quantification, concluding that damping might suffer an increase with damage on CFRPs. Based on the observation that modal damping is a parameter with higher sensitivity to internal delamination on CFRPs than natural frequencies, Keye et al. [14] develop a method using a forward approach in which the experimental and analytical damping deviations are correlated through a modified form of the Modal Assurance Criterion (MAC). Kyriazoglou et al. [15] explore the use of the Specific Damping Capacity (SDC) for damage detection and localization in composite laminates. One important observation these authors provide is that the resonant frequency did allow detecting cracks in Glass Fibre Reinforced Plastic (GFRP) laminates while, for CFRPs, no detectable changes in the resonant frequencies could be found; however, even for 
CFRPs, high changes were found in the SDC. Yam et al. [16] noted that the energy dissipation in a CFRP is mostly induced by the interfacial slip across the delamination and the tendency for mutual penetration between the upper and lower surfaces in the delamination region.

Most of the traditional methods are based on the fact that damage leads to appreciable reduction in the stiffness of a structural element. Nevertheless, in structures made of composite materials there seems to be a tendency for the use of damping as the damage feature, once damping variations - associated with the dissipated energy - tends to be more sensitive to damage than the stiffness variations, mainly when delamination is concerned.

Instrumentation is another aspect that must be taken into account apart from the features to be measured for damage assessment, as several methods tend to rely on the use of a huge amount of sensors.

The proposed technique is mainly based on the mode shapes and the variation of the modal damping factors between the undamaged and damaged states of the structure. It is also one of the purposes of this technique that measurements are carried out with conventional "low-cost" transducers (in theory, only one force and one response transducers are needed).

In summary, an inverse model-based approach for the experimental localization of damage in a CFRP plate using as main feature the modal damping factor is presented. Some numerical simulations as well as one experimental example are presented for assessment of the method.

\section{The Damping Damage Indicator (DaDI)}

Contrary to what happens in a crack, when a delamination or debonding failure mode is concerned, friction between the interacting surfaces may occur for small bending deformations. Thus, since friction is an energy dissipation mechanism, it is reasonable to assume that damping may be used as a parameter for SHM, when this type of damage is concerned. In such a case, the material is locally heterogeneous and damping should suffer an increase dependent on the deformation shape. The method presented herein is based on the premise that different mode shapes have different sensitivities at the locations where damage with a delamination is present. This principle is somehow similar to the one used in others methods such as the Mode Shape Curvature (MSC) or the Mode Shape Slope (MSS) [17, 18].

By combining the use of Finite Element Analysis (FEA) with Updating techniques, it is possible to obtain, with a reasonable degree of accuracy, a spatial description of the mode shapes and other physical 
quantities along the structure (such as strains, stresses, etc.). In the case of a thin plate, there will only be two bending planes and both the length $(x)$ and width $(y)$ directions have to be considered. Thus, a Plane Shape Function (PSF) is defined for mode $r$ at a discretization node with co-ordinates $(i, j)$ as

$$
P S F_{i j}^{r}=\frac{\left|X_{i j}^{r}\right|}{\max \left|X_{i j}^{r}\right|}+\frac{\left|Y_{i j}^{r}\right|}{\max \left|Y_{i j}^{r}\right|}
$$

where $X$ and $Y$ are two arbitrary mode dependent physical quantities in the directions $x$ and $y$ respectively. Considering that a delamination only leads to an increase in the damping factor $\eta$, the discrepancy between the undamaged and damaged state of the modal damping may be computed by

$$
\Delta \eta_{r}^{D}=\frac{\eta_{r}^{D}-\eta_{r}}{\eta_{r}}
$$

where the superscript $D$ stands for damage. It is assumed that the mode shapes and the natural frequencies will not suffer a considerable change between the undamaged and damaged states of the structure.

Consequently, the PSF will also remain essentially unchanged. Then, an index, called Damping Damage Indicator (DaDI), can be defined as

$$
D a D I_{i j}=\frac{\sum_{r=1}^{N}\left(P S F_{i j}^{r} \cdot \Delta \eta_{r}^{D}\right)}{\sum_{r=1}^{N} P S F_{i j}^{r}}
$$

For the application of equation (3), only the damping factors for both the damaged and undamaged structure are required in addition to the PSF.

Replacing $X$ and $Y$ in equation (1) by the strains ${ }_{x} \varepsilon$ and ${ }_{y} \varepsilon$ respectively, as strains are functions of both the geometry of the structure and the material properties, the PSF is given by

$$
P S F_{i j}^{r}=\frac{\left|{ }_{x} \varepsilon_{i j}^{r}\right|}{\left.\max \right|_{x} \varepsilon_{i j}^{r} \mid}+\frac{\left|{ }_{y} \varepsilon_{i j}^{r}\right|}{\left.\max \right|_{y} \varepsilon_{i j}^{r} \mid}
$$

In this case, the DaDI given by equation (3) will be a function of both the modal strain shapes and modal damping factors variation.

\section{Procedural Guidelines}

\subsection{Structural Components}


Composite structures are usually low in weight when compared to metallic alloys. In the proposed technique, one force and one response transducers must be used so that at least one point Frequency Response Function (FRF) may be extracted. Unless a hammer is used to introduce the input excitation force and non-contact transducers are used to measure the output response (such as a LASER Vibrometer), the mass per unit area of a transducer may be relatively high when compared to the mass per unit area of a composite panel. Also, for lightweight structures, the connecting wires and cables may have an influence on the results that is difficult to predict. Hence, it is recommended that attached transducers are treated as part of the structure, thus avoiding the use of mass cancelling procedure techniques which may increase the uncertainty of the results.

\subsection{Extracting Mode Shapes and Modal Damping Factors from the Structure}

In order to obtain a spatial description of the mode shapes of the structure, one of the following approaches can be used:

- A scanning LASER vibrometer, or similar device, is used to measure the responses in several nodes to a single-point excitation. Based on the out-of-plane displacements, the strains can be estimated using numerical methods;

- $\quad$ A Finite Element Model (FEM) of the structure can be used alternatively.

If a FEM is chosen, it is advisable to resort to experimental data for Updating, which means that both approaches can be used together.

In order to identify the modal damping of both the undamaged and damaged states of the structure a modal identification technique is used based on measured FRFs. In the present case, the modal damping was thereafter identified using BETAlab software that makes use of the Characteristic Response Function (CRF) $[19,20]$, but any other suitable tools can be used instead.

\section{Specimen Used to Determine the DaDI}

In order to determine the DaDI, numerical and experimental tests have been carried out. The specimen considered is a quasi-isotropic CFRP rectangular plate with a $(0,45,135,90)_{\mathrm{S}}$ lay-up and approximate dimensions of $2 \times 241 \times 390 \mathrm{~mm}$. Each layer is a woven carbon fibre prepreg constituted by Hexcel ${ }^{\circledR}$ G803 satin carbon fibre in HexPly 200 phenolique matrix (200/40\%/G803). 
It is considered that the force transducer, with an active mass of $18 \mathrm{~g}$ (total mass is $21 \mathrm{~g}$ ) and moments of inertia $\mathrm{I}_{\mathrm{x}}=\mathrm{I}_{\mathrm{y}}=1.33 \times 10^{-6} \mathrm{kgm}^{2}$ and $\mathrm{I}_{\mathrm{z}}=8 \times 10^{-7} \mathrm{kgm}^{2}$, is part of the structure.

The plate was modelled in ANSYS using a total of 1750 Shell63 elements, with 6 dof per each of the 4 nodes in the element and, for the damage localization, 6 regions have been considered, as shown in figure 1. Each of the 6 damage regions has 16 elements, corresponding to an area of approximately $1 \%$ of the total area of the plate.

Experimentally, damage was introduced in location A1 only, but two succeeding scenarios of damage were considered. In the first scenario, damage was introduced using an impact hammer from IMATEK. In the second scenario, the existing damage was increased by quasi-static loading.

\section{Numerical Examples}

Nine numerical simulations have been performed in order to study different damage scenarios. The first 6 are single damage simulations in each of the locations A1 to A6. The other 3 simulations include multiple damage locations (only damage in 2 different locations was studied). Damage was characterized by a local damping factor variation ${ }^{\dagger}$ by setting a different damping factor to each of the Shell63 elements, providing a global variation in the modal damping factors of the same order of magnitude of the experimental results (its local value was increased 100 times so that modal damping factor variations up to $200 \%$ could be found).

A total of 18 mode shapes has been considered in this study, in the range of 0 to $800 \mathrm{~Hz}$. The probability for the location of the damage is shown by the values of the DaDI along the plate (figure 2). Areas with a higher DaDI value are more likely to be damage locations.

The nine snapshots taken and shown in figure 2 show the DaDI along the plate for a threshold level of $10 \%$ (only $10 \%$ of the total area of the plate is shown, corresponding to the highest values of DaDI). It may be observed that, in the studied examples, false-positives are more likely to occur rather than falsenegatives, but in general the results are satisfactory, since DaDI is a probability indicator and shows a high value at the effective locations.

\section{Experimental Examples}

\footnotetext{
Frequency Independent (ANSYS).
} 
For experimental assessment of the proposed technique, 2 experimental situations have been studied ${ }^{\star}$ :

- "Light" damage on location A1: damage introduced by impact. (A1H-A1D);

- "Heavy" damage on location A1: previous damage introduced by impact followed by quasistatic pressure (A1D-A1DD).

In the first case, measurements were carried out in the 0 to $400 \mathrm{~Hz}$ frequency range, thus leading to the modal identification of 8 modes only. In the second case measurements were carried out up to $800 \mathrm{~Hz}$ and 18 modes have been identified.

\subsection{Test Setup}

A Pulse system from Brüel \& Kjær (B\&K), including a signal acquisition module (B\&K type 3109) and LAN interface module (B\&K type 7533), was used for both the signal generation and acquisition.

The plate was suspended in a free-free configuration using two nylon strings. One multisine excitation force was introduced at the top-left corner of the plate using an electromagnetic shaker (B\&K type 4809) and stinger. This force was measured using a force transducer (B\&K type 8200) with charge amplifier (B\&K type 2706). Four responses were measured using a dual-channel LASER vibrometer from Polytec (interferometer OFV 508 and controller OFV 2802i) and target reflective tape from 3M.

Except for the direct FRF, all the other response measurement locations were chosen so that they would not be over a nodal line of the plate up to $400 \mathrm{~Hz}$, as shown in figure 3 . These nodal lines were obtained for validation of the FEM, using two scanning LASER vibrometers from Polytec. In the first run (up to $400 \mathrm{~Hz}$ ), for condition A1H ("light" damage reference case), a combintation of head OFC-056, controller OFV-3001 and junction box PSV-Z-040M was used. In the second run (up to $800 \mathrm{~Hz}$ ), for condition A1D ("heavy" damage reference case) a combination of head PSV-400, controller OFV-5000 and junction Box PSV-400 was used.

\subsection{Some Considerations on the Damping Variation}

Until now, it has been considered that the typical form of damage in a composite material is a delamination. Furthermore, it was considered that this type of damage would lead to an increase in the

\footnotetext{
For the sake of simplicity, the several conditions of the structure will be referred to as A1H (for the health state), A1D (for the damage state introduced by impact) and A1DD (for the damage state introduced by both impact and quasi-static loading).
} 
damping as a consequence of the energy loss due to friction. Nevertheless, another typical failure mode is fibre breakage in the opposite face of the impact. In such a case, and if no significant delamination has occurred, is it possible that the damping will decrease instead?

A hint may come from Hadi and Ashton [21] who experimentally determined the material damping of several GFRP specimens showing that the damping factor is higher at low fibre volume fractions and that it increases with increasing fibre orientation up to $30^{\circ}$. The damping factor decreases as the fibre orientation increases above $30^{\circ}$. According to these authors, this happens because the total strain energy is dominated by the in-plane shear strain energy, and this has its maximum value at this fibre orientation. Thus, if a fibre breakage occurs in a specimen with no delamination, than it is reasonable to assume that, for some mode shapes, damping will decrease.

In order to obtain some insight, it was decided to plot the damping variation $\Delta \eta_{r}^{D}$ with respect to the mode number, for both the experimental cases (figure 4).

The $\Delta \eta_{r}^{D}$ is negative for some modes (dashed line). For instance, mode 2 has a negative value in both situations, whereas mode 4 has a negative vale for situation A1H-A1D and a positive value for situation A1D-A1DD. However, the negative values are generally low when compared to other values in the whole domain. Since these results are not enough to draw a reliable conclusion so far, it was considered that, due to the well known difficulty on estimating the modal damping with enough accuracy, the lower damping values might be a consequence of uncertainty. Thus, the following correction has been made to the values of $\Delta \eta_{r}^{D}$ in the procedure:

$$
\left|\Delta \eta_{r}^{D}\right| \leq \min _{r}\left(\Delta \eta_{r}^{D}\right) \mid \Rightarrow \Delta \eta_{r}^{D}=0
$$

In this way, the modes that have a damping variation $\left|\Delta \eta_{r}^{D}\right|$ lower than the absolute value of the minimum $\Delta \eta_{r}^{D}$ are not considered as being sensitive to local damage. The effects of this correction on the damping factor variations $\Delta \eta_{r}^{D}$ can be observed in figure 4 (continuous line) $)^{\S}$.

\subsection{Results}

\footnotetext{
$\S$ This correction was not made for the numerical examples, since damage was simulated by a local increase of the damping. Thus, $\Delta \eta_{r}^{D}$ remained positive for all the modes.
} 
The DaDI values along the plate are shown in figure 5. For the A1H-A1D case, the damage is not clearly identified and the presence of the force transducer seems to introduce a high influence on the results. It must be pointed out that the force transducer weighs $21 \mathrm{~g}$, which represents $7 \%$ of the total weight of the structure (296g). However, the damage is still located close to a high DaDI values area. In the second example, A1D-A1DD, for a heavier damage and in which higher order mode shapes were included, the DaDI points closer to the damage location. In fact, the two highest values of the DaDI occur within the "bean shaped" damage probability location area, one of each is precisely at the co-ordinates of impact.

Comparison of situations A1H-A1D and A1D-A1DD raises the question of what is influencing more the results: the damage severity or the number of available mode shapes? To answer this question, or at least to have a better insight on the influence of the number and type of mode shapes, the DaDI for situation A1D-A1DD was recalculated, using modes 1 to 8 separated from modes 9 to 18 , as shown in figure 6 . Observation of these pictures shows that not only the amount of available modes is important, but also the lower order modes are necessary in damage localization techniques. In fact, while using the same amount of modes, cases A1H-A1D and A1D-A1DD are not very different from each other with respect to the damage localization, although the probability area is sparse. Both cases highlight the presence of the force transducer. On the other hand, when used separately, higher order modes tend to provide several falsepositives. In summary, it can be said that lower order modes provide a fuzzy probability distribution of the damage location whereas higher order modes sharpen the damage location prediction.

\subsection{Some Considerations on the Frequency Variation}

It is reasonable to assume that fibre breakage locally reduces the stiffness of the component and thus a shift on the natural frequencies may happen. Nevertheless, they will not be as evident as changes in damping caused by interlaminar friction, when a delamination is present. In the damage case A1D-A1DD the variation of both the natural frequencies and modal damping factors were plotted in order to evaluate whether the frequency would suffer a significant increase in the presence of damage (figure 7). It can be seen that, when compared to damping, the relative frequency shifts are very low. In this particular case, the highest frequency shift observed was a decrease of $1.3 \%$ only, whereas the highest modal damping factor variation showed an increase of $163 \%$. 


\section{Conclusions}

A novel method for the localization of delamination damage in composite materials has been presented, using conventional, "low-cost" and generally easy to obtain equipment. This method uses the modal damping factor variation from a reference state to a damaged state as feature. It also makes use of the modal strain shapes, described by what is called a Plane Shape Function (PSF), as a tool to spatially describe the local sensitivity to damage. The damage location is assessed by an index, called the Damping Damage Indicator (DaDI), which provides a geometrical probability indication of the damage location. It is possible to highlight the following advantages of this method:

- In theory, only one response transducer may be used (more are required to avoid modal nodes and to reduce the uncertainty);

- It is applicable at low-cost to structures that have not been previously instrumented;

- So far, it provides a probability geometrical description of the damage location for any bidimensional structure.

As disadvantages, the following can be pointed out:

- It either requires a reliable FEM (Updating is desirable) or experimentally measured mode shapes;

- As other related methods, its success is strongly dependent on the amount (and type) of mode shapes used;

- It is neither false-positive nor false-negative free, although false-positives are more likely to occur.

Further research is still required, however, in order to improve the sensitivity of the method and avoid both false-positives and false-negatives. Although not explored in this paper, it is the belief of the authors that further developments of this technique will allow to both provide a measure of the severity of damage and to distinguish between delamination and fibre breakage in CFRPs.

\section{Acknowledgments}

The authors gratefully acknowledge Prof. Patrick Guillaume and his Ph.D. student Christof Devriendt from the Faculty of Applied Sciences of the Vrije Universiteit Brussel for the use of their laboratory 
equipment in their facilities, in particular two scanning LASER vibrometers, as well as giving valuable advices and guidance in the experimental testing.

Thanks are also due to Prof. João Travassos and Paulo Caldeira from Instituto Superior de Engenharia de Lisboa of the Instituto Politécnico de Lisboa for the use of their autoclave and consumables for the production of CFRP plates, as well as for all the time they spent helping us during that process, as well as to Prof. Rui de Carvalho from Instituto Superior Técnico of the Universidade Técnica de Lisboa and Marco Leite from Escola Superior de Tecnologia de Abrantes of the Instituto Politécnico de Tomar for the help and expertise on the introduction of BVID impact damage in the CFRP laminates. Finally, the authors would like to acknowledge the financial support of Fundação para a Ciência e Tecnologia.

\section{References}

[1] B. Bonfiglioli, A. Strauss, G. Pascale, K. Bergmeister, Basic Study of Monitoring on Fibre Reinforced Polymers: Theoretical and Experimental Study, Smart Materials and Structures 14 (2005) 12-23.

[2] S.W. Doebling, C.F. Farrar, M.B. Prime, D.W. Shevits, Damage Identification and Health Monitoring of Structural and Mechanical Systems from Changes in their Vibration Characteristics: A Literature Review, in: Report to the Los Alamos National Laboratory, Los Alamos, USA, 1996.

[3] S.W. Doebling, C.R. Farrar, M.B. Prime, A Summary Review of Vibration-Based Damage Identification Methods, Shock and Vibration Digest 30 (2) (1998) 91-105.

[4] H. Sohn, C.R. Farrar, F.M. Hemez, D.D. Shunk, D.W. Stinemates, B.R. Nadler, A Review of Structural Health Monitoring Literature: 1996-2001, in: Report to the Los Alamos National Laboratory, Los Alamos, USA, 2003.

[5] D. Montalvão, N.M.M. Maia, A.M.R. Ribeiro, A Review on Vibration Based Structural Health Monitoring with Special Emphasis on Composite Materials, Shock and Vibration Digest 38 (4) (2006) 295-324.

[6] G. Park, H. Sohn, C.R. Farrar, D.J. Inman, Overview of Piezoelectric Impedance-Based Health Monitoring and Path-Forward, Shock and Vibration Digest 35 (6) (2003) 451-463.

[7] E.P. Carden, P. Fanning, Vibration Based Conditioning Monitoring: A Review, Structural Health Monitoring. 3 (4) (2004) 355-377. 
[8] T. Uhl, K. Mendrok, Overview of Modal Model based Damage Detection Methods, in: Proceedings of the ISMA 2004 International Conference on Noise and Vibration Engineering, Leuven, Belgium, 2004, pp. $361-375$.

[9] C.R. Farrar, H. Sohn, A.N. Robertson, Applications of Nonlinear System Identification to Structural Health Monitoring, in: Proceedings of the Second European Workshop on Structural Health Monitoring, Munich, Germany, 2004, pp. 59-67.

[10] D.R. Sanders, Y.I. Kim, N. Stubbs, Nondestructive Evaluation of Damage in Composite Structures Using Modal Parameters, Experimental Mechanics 32 (3) (1992) 240-251.

[11] F.L. Matthews, Damage in Fibre Reinforced Plastics; Its Nature, Consequences and Detection, in: Proceedings of the DAMAS99 Third International Conference on Damage Assessment of Structures, Dublin, Ireland, 1999, pp. 1-16.

[12] R.J.S. Carvalho, Resistência Residual de um Compósito de Matriz Epóxídica reforçado por Fibras de Carbono após Reparação, Ph.D. Thesis, Instituto Superior Técnico, Technical University of Lisbon, Lisbon, Portugal, 2003 (in Portuguese).

[13] P. Cawley, R.D. Adams, The Location of Defects in Structures from Measurements of Natural Frequencies, Journal of Strain Analysis for Engineering Design 14 (2) (1979) 49-57.

[14] S. Keye, M. Rose, D. Sachau, Localizing Delamination Damages in Aircraft Panels from Modal Damping Parameters, in: Proceedings of the IMAC XIX International Modal Analysis Conference, Kissimmee, Florida, USA, 2001, pp. 412-417.

[15] C. Kyriazoglou, B.H. Le Page, F.J. Guild, Vibration Damping for Crack Detection in Composite Laminates, Composites, Part A: Applied Sciences and Manufacturing 35 (2004) 945-953.

[16] L.H. Yam, Z. Wei, L. Cheng, Nondestructive Detection of Internal Delamination by Vibration-based Method for Composite Plates, Journal of Composite Materials 38 (24) (2004) 2183-2198.

[17] O.S. Salawu, C. Williams, Damage Location using Vibration Mode Shapes, in: Proceedings of the IMAC XII International Modal Analysis Conference, Honolulu, Hawaii, USA, 1994, pp. 933-939.

[18] Y.K. Ho, D.J. Ewins, On the Structural Modal Identification with Mode Shapes, in: Proceedings of the European COST F3 Conference on System Identification and Structural Health Monitoring, Madrid, Spain, 2000, pp. 677-686.

[19] N.M.M. Maia, J.M.S. Silva, A.M.R. Ribeiro, A New Concept in Modal Analysis: The Characteristic 
Response Function, The International Journal of Analytical and Experimental Modal Analysis 9 (3) (1994) 191-202.

[20] J.M.M. Silva, N.M.M. Maia, A.M.R. Ribeiro, Structural Dynamic Identification with Modal Constant Consistency using the Characteristic Response Function (CRF), Machine Vibration 5 (2) (1996) 83-88.

[21] A.S. Hadi, J.S. Ashton, Measurement and Theoretical Modeling of the Damping Properties of a UniDirectional Glass/Epoxy Composite, Composite Structures 34 (1996) 381-385. 


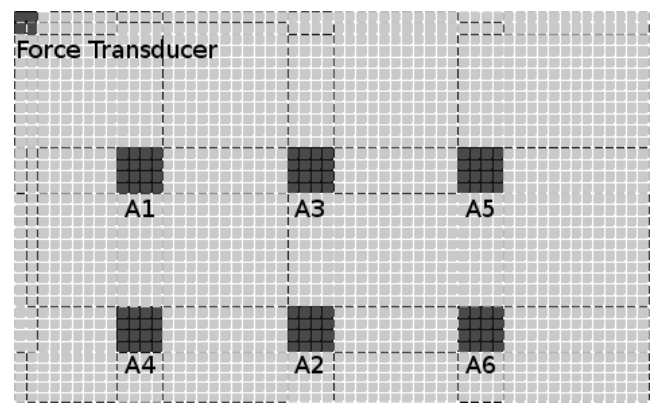

Fig. 1 - FEM of the studied plate: force transducer and damage locations. 


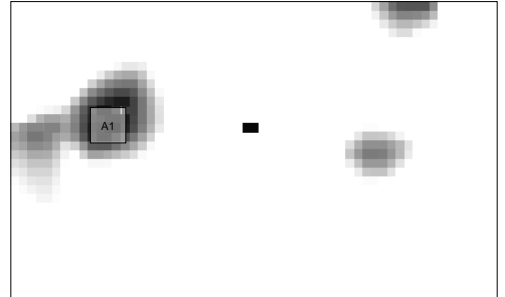

Damage Location: A1

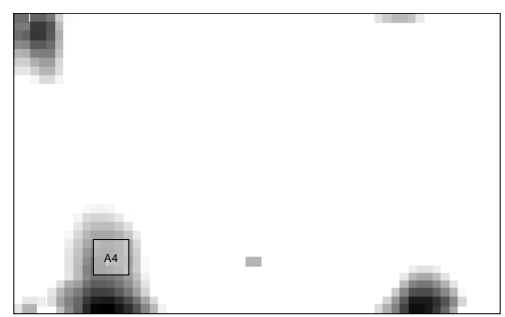

Damage Location: A4

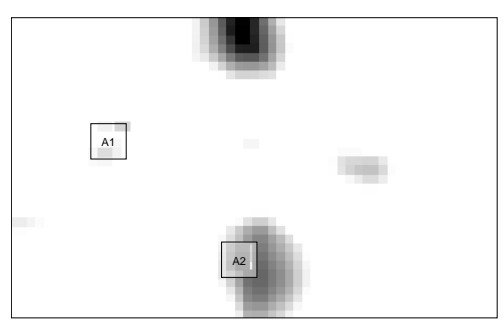

Damage Locations: A1 and A2 Lower DaDI

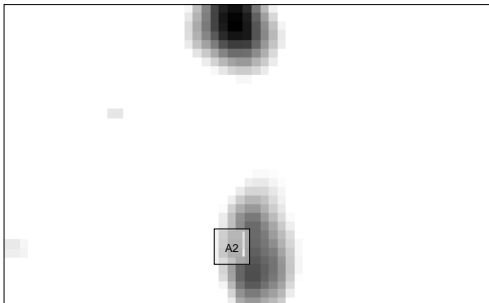

Damage Location: A2

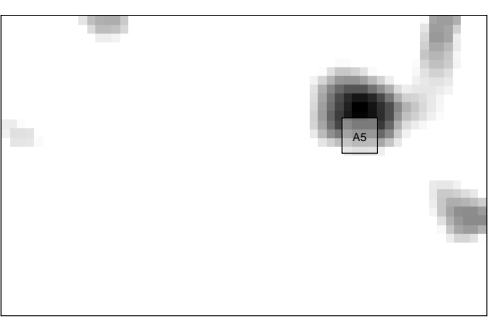

Damage Location: A5

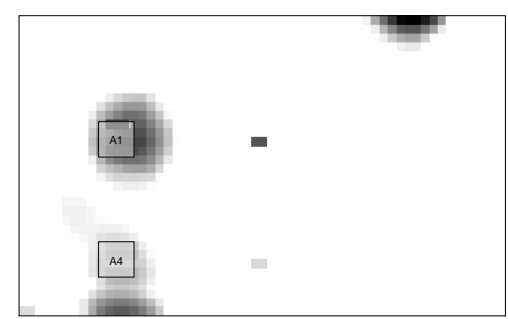

Damage Locations: A1 and A4

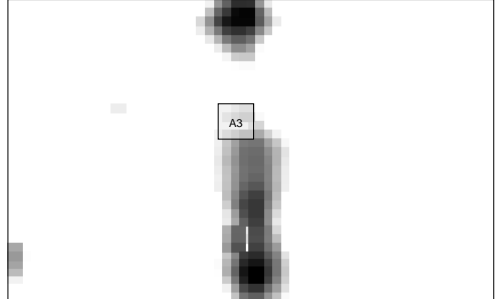

Damage Location: A3

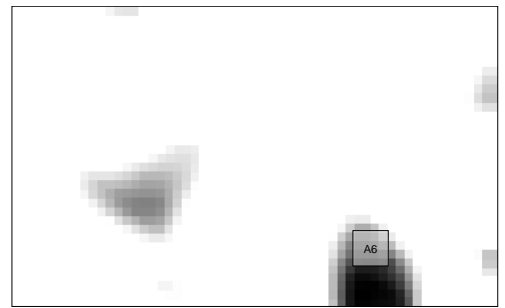

Damage Location: A6

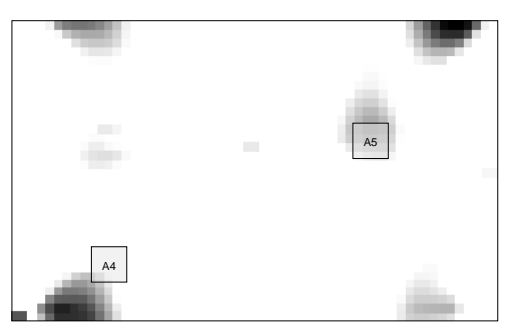

Damage Locations: A4 and A5 Higher DaDI

Fig. 2 - DaDI probability along the plate: numerical examples (area threshold level $\approx 10 \%$ ). 


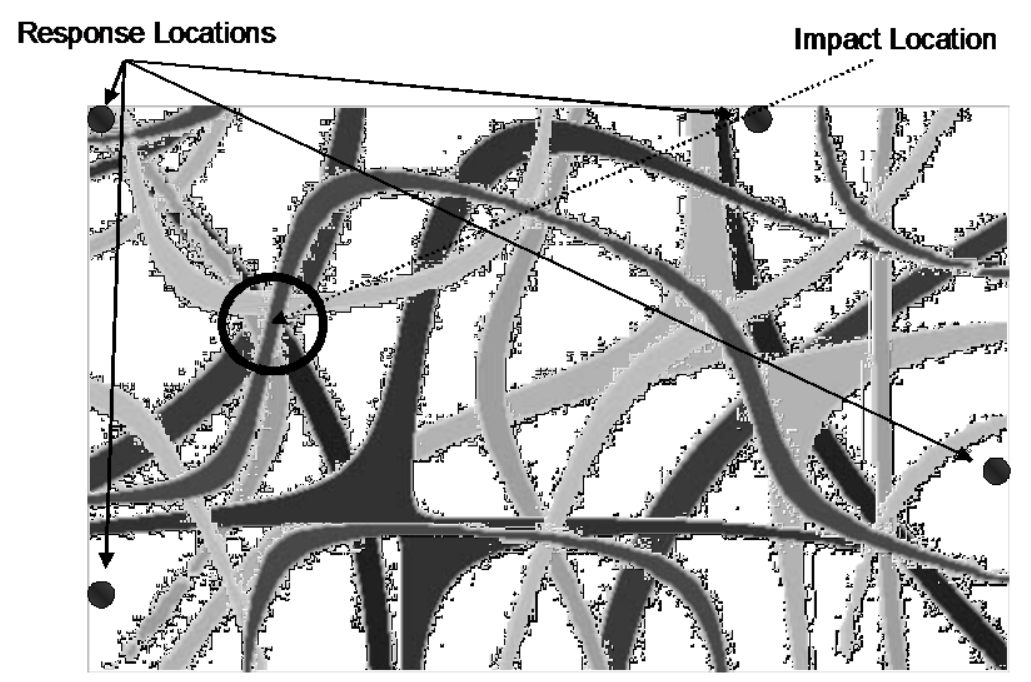

Fig. 3 - Measurement locations for the responses, experimental damage location and nodal lines of the plate until the $400 \mathrm{~Hz}$ frequency range (8 modes visible). 


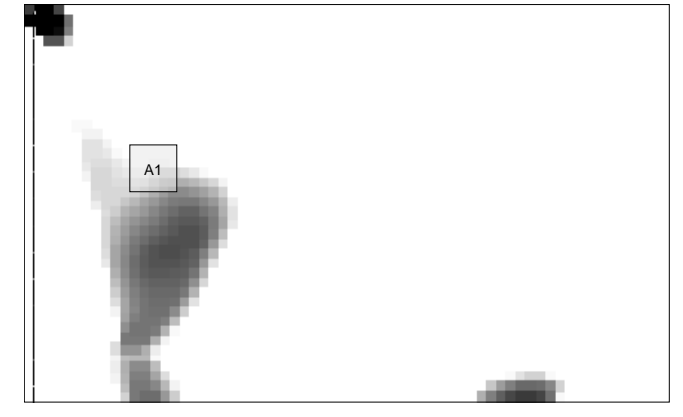

A1H-A1D Damage Case 8 modes, $0-400 \mathrm{~Hz}$

Lower DaDI

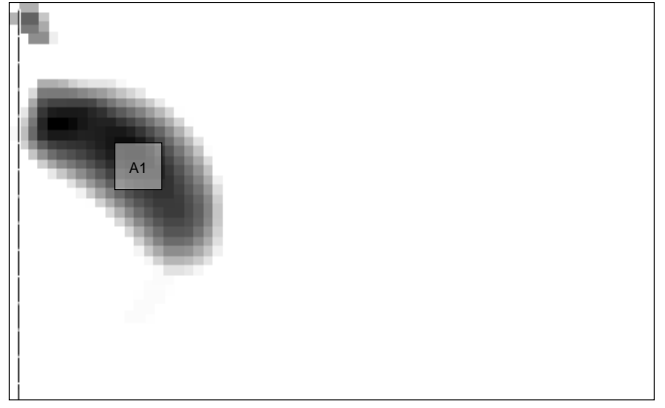

A1D-A1DD Damage Case

18 modes, $0-800 \mathrm{~Hz}$

Higher DaDI

Fig. 4 - DaDI probability along the plate: experimental examples (area threshold level $\approx 10 \%$ ). 


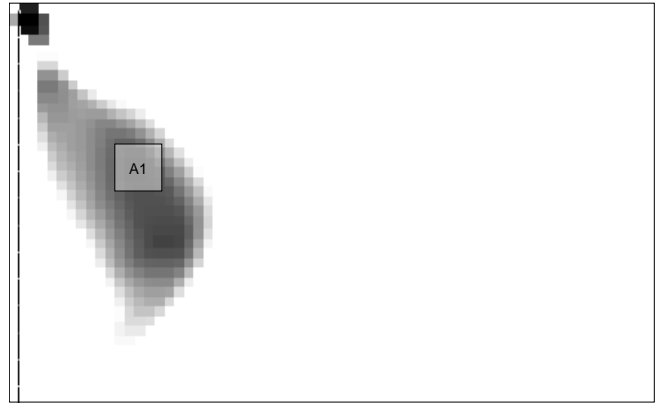

A1D-A1DD Damage Case Modes 1 to $8,0-400 \mathrm{~Hz}$

Lower DaDI

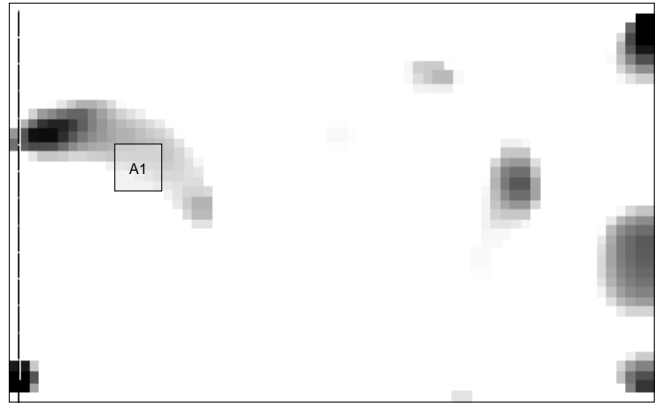

A1D-A1DD Damage Case Modes 9 to $18,400-800 \mathrm{~Hz}$

Higher DaDI

Fig. 5 - DaDI probability along the plate: experimental example A1D-A1DD for lower order modes (left) and higher order modes (right) (area threshold level $\approx 10 \%$ ). 
“Light” Damage Case - A1H-A1D

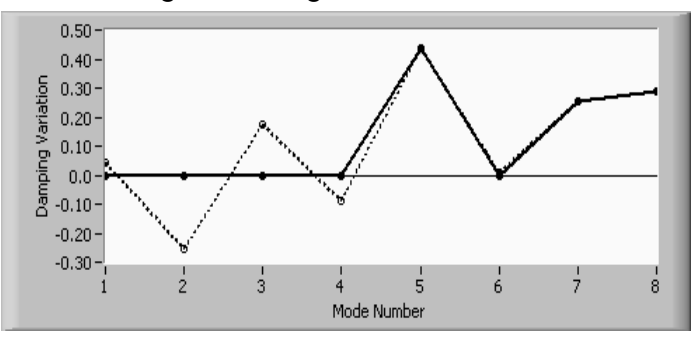

"Heavy" Damage Case - A1D-A1DD

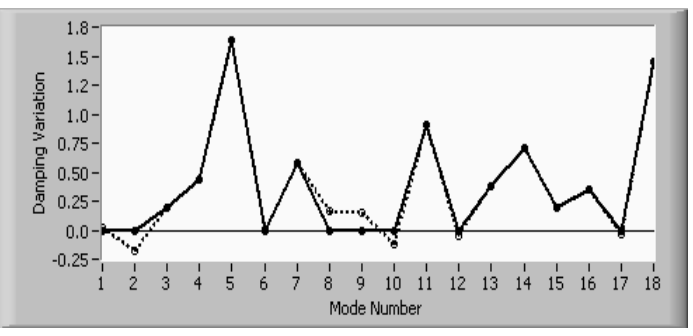

Fig. 6 - Damping variation $\Delta \eta_{r}^{D}$ (dashed line) and correction (continuous line) with respect to the mode number for cases A1H-A1D (left) and A1D-A1DD (right). 


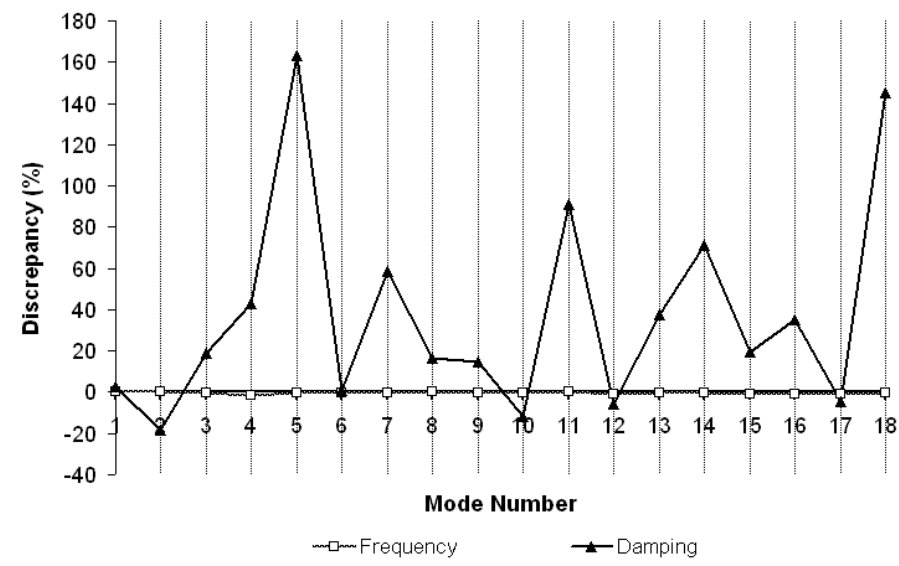

Fig. 7 - Natural frequency and modal damping factor shifts due to the presence of damage for the experimental case A1D-A1DD. 